\title{
LA PULSION DE MORT À L'ÉPREUVE DU MATERNEL
}

Michèle Benhaïm

\author{
ERES | «Analyse Freudienne Presse »
}

2002/2 n 6 | pages 101 à 113

ISSN 1253-1472

ISBN 2749200180

Article disponible en ligne à l'adresse :

http://www.cairn.info/revue-analyse-freudienne-presse-2002-2-page-101.htm

\section{Pour citer cet article :}

Michèle Benhaïm, «La pulsion de mort à l'épreuve du maternel », Analyse Freudienne Presse 2002/2 (n 6), p. 101-113.

DOI 10.3917/afp.006.0101

Distribution électronique Cairn.info pour ERES.

(c) ERES. Tous droits réservés pour tous pays.

La reproduction ou représentation de cet article, notamment par photocopie, n'est autorisée que dans les limites des conditions générales d'utilisation du site ou, le cas échéant, des conditions générales de la licence souscrite par votre établissement. Toute autre reproduction ou représentation, en tout ou partie, sous quelque forme et de quelque manière que ce soit, est interdite sauf accord préalable et écrit de l'éditeur, en dehors des cas prévus par la législation en vigueur en France. Il est précisé que son stockage dans une base de données est également interdit. 


\section{Michèle Benhaïm}

\section{La pulsion de mort à l'éprenve du maternel}

Si l'enfant paraît, c'est qu'il est peut-être au commencement " pure pulsion de vie ". Ce titre La pulsion de mort à l'épreuve du maternel est une façon de poser une question : Qu'advient-il de la pulsion de mort de la mère ? quel traitement subit-elle au cours de la traversée du processus de maternité ? Alors que nous la savons "silencieuse ", "muette ", faisant régner le silence dans le "ça ", comment s'exprime-t-elle, et comment s'élabore-t-elle ? Cette question trouve sa légitimité si l'on considère que la pulsion de mort est convoquée à chaque coupure jalonnant le parcours maternel (division cellulaire, accouchement, sevrage, adolescence). Coupures réelles tout autant que symboliques si l'on pense que chacun de ces passages repose sur une alternative vie/mort.

Façon de s'inscrire dans la conception dualiste freudienne : «Nous avons pris pour point de départ l'opposition

Michèle Benhaïm, psychanalyste, Marseille. 
entre les instincts de vie et les instincts de mort. L'amour concentré sur un objet nous offre lui-même une autre polarité de ce genre : amour proprement dit (tendresse) et haine (agression) ${ }^{1}$ ». Et Freud d'ajouter ce que je reprends aujourd'hui totalement à mon compte : "Si seulement nous pouvions réussir à établir un rapport entre ces deux polarités, à ramener l'une à l'autre!"

Façon également de rappeler le lien étroit qui unit la pulsion de mort au langage, c'est-à-dire sa situation entre le sujet et l'Autre. Impliquant de la sorte la mère comme première incarnation de l'Autre.

\section{LA HAINE}

Si la haine ne recouvre pas purement et simplement la pulsion de mort, elle peut en être une traduction. L'hypothèse serait qu'ainsi convoquées chez la mère, à chaque nouvelle confrontation à la coupure, les pulsions se désintriqueraient et la pulsion de mort pourrait alors connaitre deux destins : soit avoir un effet dans le réel portant atteinte directe au corps de l'enfant, voire à sa vie ; soit demeurer un des termes de la vie psychique, certes, mais déjà aussitôt réintriquée à la pulsion de vie, déjà symbolisée, déjà nommée, pourrait-on dire. Autrement dit, elle pourrait, dans ce cas de figure, le plus fréquent bien sûr, prendre les traits de la haine, non pas destructrice et mortelle, mais haine nécessaire, vitale, structurante en ce qu' elle limiterait, diviserait, barrerait, poserait une condition à l'amour maternel, primordial, tout-puissant dont on sait les ravages.

Cette haine nécessaire, symbolique et non pas imaginaire, pourrait, en installant la mère dans une ambivalence du sen- 
timent, faire que se pacifient les pulsions de vie et les pulsions de mort, quand la question de la vie et de la mort se pose.

Je vais donc tenter d'exposer quelques idées autour de l'articulation de la pulsion de mort avec l'ambivalence de la mère, et de montrer quelques occurrences cliniques dans lesquelles des pathologies graves de l'enfant relèvent de drames parce que la pulsion de mort à l'œuvre dans le réel se loge au cœur de la relation fondatrice. Je pense notamment à l'anorexie mentale nouée dans les premiers échanges mère/enfant et à l'infanticide.

En 1915, Freud affirme: "La fin vers laquelle tend toute vie est la mort et inversement, le non-vivant est antérieur au vivant ", s'appuyant entre autres sur des propos de Goethe (1883) pour qui «la mort est une conséquence directe de la procréation ». Si Freud fait de la pulsion de mort la pulsion par excellence en vertu de son caractère répétitif et régressif, il se demande néanmoins « comment déduire d'Éros, dont la fonction consiste à conserver et à entretenir la vie, cette tendance sadique à nuire à l'objet ". Si on continue non pas à les superposer, mais à tenter d'articuler les doubles polarités, on peut en écho se demander comment déduire de l'amour la haine. Freud continue : "Le sadisme est un instinct de mort que la libido narcissique a détaché du moi et qui trouve à s'exercer sur l'objet. " En effet, lorsque la pulsion de mort s'exprime en se tournant vers l'extérieur, elle est pulsion de destruction. Nous pouvons ajouter : l'amour coïncide avec la destruction de l'objet.

Avant même son travail sur les névroses de guerre et son observation de la compulsion de répétition, notamment au travers du Fort-Da, deux pistes qui le mettent sur la voie de la pulsion de mort, Freud avait vu dans la haine une relation aux objets plus ancienne que l'amour. Il posait alors une sorte d'impossibilité, d'un point de vue métapsychologique, de déduire cette haine des pulsions sexuelles. Puis, en introduisant le narcissisme, moment où, pour lui, cessent de s'oppo- 
ser pulsions sexuelles et pulsions du moi pour devenir des modalités de la libido, il se trouve obligé de proposer un autre dualisme : pulsions de vie/pulsions de mort. En modifiant son dualisme des pulsions et en proposant l'hypothèse de l'existence d'une pulsion de mort, Freud remet en question le primat du principe de plaisir. Il avance dans le même cheminement : ce n'est ni de la haine, ni de l'agressivité ou même de l'ambivalence que se déduit l'existence de la pulsion de mort. Pour cette raison, si on ne peut substituer la haine à la mort ou encore un sentiment à une pulsion, on notera néanmoins que Freud conserve la polarité de l'amour et de la haine pour illustrer la polarité des deux pulsions (Éros et Thanatos) dans la mesure où, la pulsion de mort étant "muette ", nous n'y avons par définition accès qu'à partir de ses manifestations dirigées vers l'extérieur. Il la nomme alors pulsion de destruction.

\section{PULSION DE DESTRUCTION}

Si la haine initiale est alors une des figures possibles de la pulsion de mort, mon hypothèse est qu'elle sera d'autant plus violente et meurtrière qu'elle aura été initialement évacuée, mise hors d'état d'être pensée.

Dans le processus maternel se font jour les effets de la pulsion de mort sur le rapport de la mère à son objet d'amour, l'enfant. Dès l'origine, la pulsion de mort devient perceptible pour le sujet. Nous l'avons dit, aimer un objet, c'est aussi le détruire.

En psychopathologie, si nous n'avons, par définition, pas accès directement à la pulsion de mort (encore que Freud ait décrit la seule occurrence où elle se laisse entrevoir à l'état pur, le mélancolique dont le surmoi apparât, dit-il, comme une "pure culture de la pulsion de mort ${ }^{2}$ »), on peut avoir à 
en entendre ou à en voir les effets qui en dérivent. Ils prennent les traits de la pulsion d'agression comme dans le caprice maternel où nous voyons l'agressivité basculer dans la violence. Ou encore de la pulsion de destruction comme dans l'infanticide, bien sûr, ou peut-être dans ce que Serge Leclaire décrit à partir de "On tue un enfant ". Là, si nous le suivons, il n'est de vie qu'au prix du meurtre de l'image première, étrange, dans laquelle s'inscrit la naissance de chacun, meurtre nécessaire tout autant qu'irréalisable de l'enfant merveilleux en nous, naissance sur fond de meurtre du narcissisme primaire. Là, Leclaire nous indique qu'à en faire l'économie, le sujet risque de confondre cette première mort avec la seconde, la vraie, et évoque à ce propos des cas où la question retentit sur les propres enfants du sujet : morts néonatales, malformations, enfants nés avant terme, enfants brutalement et sans raison atteints très tôt de maladies gravissimes ou rares, accidents infantiles relevant d'un quasipassage à l'acte fatal. Bref, ces cas où, à défaut de résoudre la question de l'enfant à tuer, un enfant meurt dans le réel. Le fantasme de Leclaire recouvre le concept d'[enfant-mort] décrit par Jacques Hassoun, qui en fait une "figure inarticulée du vœu de la mère ", et complète les propos de Jenny Aubry sur " une mère à tuer ", chute de (a) : la mère non plus ne l'a pas.

Ces détours intéressent notre propos et me permettent d'articuler les questions autour de la pulsion de mort et de la haine initiale qui peut la traduire, à celles qui trouvent à se résoudre dans l'ambivalence maternelle (amour/haine vitale). Résolution subjectivement et réellement salvatrice pour l'enfant comme pour la mère.

Hors d'état d'être pensée, la haine en question demeure jouissance pulsionnelle qui ne passe pas par le langage, jouissance comme satisfaction de la pulsion qui échappe aux défilés du signifiant, jouissance censée être interdite à l'être parlant. La mère jouit de l'enfant dans une unité idéale. Hors 
fantasme, le caprice maternel renvoie au réel, à l'angoisse, à l'horreur. La jouissance pure, non voilée, captive l'enfant dans une sorte de complémentarité narcissique de la mère, comme lieu de projection absolu où s'inscrit la haine initiale, sans envers, comme reflet/effet de la pulsion de mort déliée ici de ce qui est censé la tapir dans le silence, la pulsion de vie. Le bébé, en refusant la séparation et la coupure du sein maternel, met en place cette unité mythique primordiale. Soumis au sevrage, de par sa prématurité biologique qui le rend absolument dépendant, et devant lâcher cet objet (a), le sein, comme une séparation d'avec une part de lui-même, il croit qu'il va mourir. Seule l'ambivalence que peut alors lui opposer la mère devenue A barré pourra l'inscrire lui aussi dans une ambivalence dialectique, coexistence contradictoire d'acceptation et de refus. Mais, à l'inverse, peut se développer ce que Lacan nomme un "véritable appétit de la mort " où le sujet, dans son abandon, cherche à retrouver un en-deçà maternel, c'est-à-dire à y disparaître, l'Autre n'étant pas encore, à ce stade, constitué comme extérieur au sujet ; or, si la mère est confondue avec un Autre non barré, l'enfant ne peut qu'être en place d'objet de son désir.

Dans ce contexte, l'enfant n'est pas un objet perdu, car il aurait alors plutôt soutenu l'amour, mais un objet réel supportant du fait de ce statut cet amour tout-puissant, inconditionnel, c'est-à-dire illimité, que Freud, Klein, Winnicott ont déjà nommé haine originaire, primordiale, initiale.

C'est cette haine-là que l'on peut dire consubstantielle à la pulsion de mort. Face à elle, Moustapha Safouan est radical, pas d'autre alternative, si ce n'est : "La parole ou la mort. »

Questionner la pulsion de mort dans son rapport au maternel soulève plusieurs remarques : si l'on parcourt l'œuvre de Freud, on s'aperçoit comment, ici et là, parce qu'il faut aller débusquer les passages où il évoque le maternel, il met le plus souvent la maternité en rapport avec la mort, dans 
un seul et même mouvement, évoquant une sorte de souhait de retour au "giron maternel ", souhait de vie de ne pas être en vie : l'enfant comme la mère souhaiterait pareillement et idéalement conserver l'enfant en soi, pour ne pas le perdre, c'est-à-dire pour ne pas s'en séparer, et dans le souhait qu'il ne lui arrive " rien ». Qu'il soit mort pour ne pas mourir.

Mère et enfant sont concernés par ce mythe de la MèreToute : l'enfant dans son mouvement de fusion mortifere avec l'imago maternelle, la mère dans son impossibilité de se confronter à la castration, confrontation mise à mal par l'état même de grossesse quand il est pris à la lettre dans son aspect de complétude.

L'union avec la mère suscite la terreur de la mort. Certains patients psychotiques nous font part parfois de l'angoisse innommable qui les saisit lorsqu'ils sont seuls avec leur mère, et Conrad Stein confirme : "Le désir, qui est d'union, peut être traduit en une expression déjà figurée par le retour dans le sein maternel. Une telle restitution d'un état antérieur à la naissance serait de non-être, elle ne peut qu'évoquer la mère. "

À la suite de Freud, Lacan montre comment "l'imago salutaire à l'origine peut devenir facteur de mort ». Il dit aussi "l'amour maternel est cause de tout... " et Jacques Hassoun aimait rappeler que lorsque Lacan employait le mot " tout ", c'est que la catastrophe était proche...

FORT-DA

Cliniquement, Freud mettra en avant la dimension mortelle en décrivant le Fort- $D a$, cette dramatisation scénique qui introduit une « liaison de la pulsion de mort à la pulsion de vie ".

Au cours du jeu, l'enfant effectue, lui, un travail qui rend possible l'élaboration psychique de la perte d'objet. Suite à une perte de soins maternels, à une perte de sa présence, 
Ernst tente une maitrise symbolique de l'objet perdu et, pour ce faire, d'objet aimé et passif de soins, il se fait actif, c'est-àdire sujet de l'appel, de la demande, se constituant ainsi comme sujet aimant. De plus, dans ce renversement dialectique qui le sauve, l'enfant entre activement dans le système signifiant jusqu'à se l'appliquer à lui même. Ernst donne corps à l'absence de l'objet en l'évoquant. Il se nomme objet perdu pour la mère.

Si cette élaboration symbolique repose sur un détour par la compulsion de répétition dans laquelle Freud décèle justement la pulsion de mort, et qui consiste à répéter le départ de la mère, l'événement douloureux, plus que son retour, provoquant le plaisir, elle n'en demeure pas moins structurante en ce que l'enfant, là, traite à travers le langage l'absence de la mère, qui dès lors ne relève plus d'un caprice mortifere mais d'un désir dans lequel l'enfant peut s'inscrire tout en s'en détachant déjà, c'est-à-dire sans y demeurer aliéné.

Nommer un objet, c'est évoquer son absence, première élaboration mentale s'effectuant dans le Fort-Da sur le meurtre de la chose et instituant de la sorte l'enfant comme sujet. Pour Lacan, "La pulsion de mort est constituante de cette position fondamentale du sujet humain. Le moment où le désir s'humanise est aussi celui où l'enfant naît au langage 3 ". Cliniquement, Lacan repérera que la mère, par le sevrage, présente à l'enfant une face de vie et une face de mort. L'anorexie du bébé signe peut-être que la seconde face peut seule être présentée à l'enfant, ce que nous pouvons faire reposer sur la haine initiale comme seule expression de l'amour, figure, de ce fait, de la seule pulsion de mort. Autrement dit, l'angoisse de l'enfant d'être assassiné par la mère que Freud repérait en 1931, d'être tué ou empoisonné, serait associée à un " avatar de la relation avec elle ", le retrait du sein. 
Peut-être pouvons-nous avancer que la pulsion de mort chez la mère trouve à s'inscrire dans son hyperprésence empêchant l'élaboration mentale, le maintien de l'objet au-delà de l'absence réelle, le langage, lui faisant, dans le même mouvement, faire l'économie de la question du manque. Il ne manque rien à personne. Est-ce ce « rien » qu'incorpore répétitivement l'anorexique?

Les fantasmes maternels des mères de nourrisson, peur de le tuer, de le faire tomber, de le jeter par la fenêtre, reflètent peut-être en écho au bébé qui confond sevrage et mort, la croyance maternelle qu'en sevrant son enfant, elle le tue. Si en général la réintrication des pulsions permet de résoudre cette question dans le fantasme dont la grammaire même (on bat un enfant) illustre comment le sujet est en effet pris dans l'intrication des pulsions, fantasme en l'occurrence de culpabilité pour la mère, son échec peut engendrer le passage à l'acte dont l'infanticide est la plus évidente des figures.

\section{AmbivalenCE}

La vie de l'enfant comme sujet parlant et désirant semble s'originer dans l'effondrement du rêve maternel. Voici autrement entrevus les deux destins possibles de la pulsion de mort maternelle : soit la vie se fonde sur le sacrifice de l'un ou de l'autre dans le réel, soit elle repose sur la mort de l'image narcissique primaire.

Tout désir et toute attente d'un enfant me semblent devoir en passer par ce retour, celui de la représentation de l'enfant du narcissisme parental. À défaut, si l'[enfant-mort] n'inaugure pas la naissance, nous voici dans l'attente d'un enfant réduite à un retour du semblable qui laisse présager d'une captivité de cet enfant dans la mort dans la réalité. Si l'on veut différencier souhaits de mort et haine, on peut supposer qu'à élaborer la seconde on contourne ce que les premiers induisent. 
L'hypothèse de la nécessité d'une ambivalence maternelle, et non l'ambivalence comme on l'entend communément, importe d'un point de vue structural, c'est-à-dire comme structurant nécessairement l'amour maternel que de la haine tempérerait pour autoriser l'enfant à se séparer de ce qui le fait vivre mais qui le ferait mourir s'il ne s'en sépare pas.

Freud dit de l'ambivalence qu'elle est l'opposition de deux tendances existantes et souvent d'" inégale " puissance. Nous insisterons sur la notion d' "inégale " : les pulsions sont convoquées lors des différentes étapes de la maternité, en l'occurrence quand quelque chose d'une séparation doit s'élaborer, en particulier à la traversée du réel que constitue l'accouchement, ce temps où du corps sort du corps. Si l'ambivalence ne relaye pas ici la question " qui des deux va survivre ? ", l'enfant peut demeurer ce réel inintégrable d'une mère qui n'aurait alors pas de choix autre que de l'abandonner ou de le vouer à la mort. Autrement dit, si la perte que constitue la mise au monde de l'enfant - rapport au réel de la mort, appréhendé côté mère et lié à sa question de la castration dans laquelle l'enfant s'inscrit -, si cette perte n'est pas aussitôt intégrée, par exemple sous la forme d'une dépression légère et temporaire, alors la pulsion de mort peut prendre le pas sur la pulsion de vie. Avoir un enfant, c'est justement ne plus l'avoir.

Ceci pose que le rapport de la mère à l'enfant est d'emblée ambivalent et recouvre ce que je situe du côté de la haine. Il est nécessaire à ce que s'élabore d'emblée la séparation, la coupure symbolique d'avec l'enfant. Chaque occurrence de coupure tout au long de la maternité met la mère en demeure de réinterroger le manque et de se repositionner dans l'énonciation de son désir.

De la même façon, la "mère suffisamment bonne " de Winnicott nous intéresse dans le "suffisamment ». Exactement là s'inscrit cette haine vitale, sinon Winnicott aurait sans doute dit la "mère bonne " et sans cet écart qu'instaure 
le "suffisamment", la mère pourrait demeurer aux prises avec l'enfant qui, d'être d'emblée radicalement autre, la prive d'une satisfaction narcissique espérée.

C'est cette haine non symbolisée en coupure qui, s'étayant sur la pulsion de mort alors dominante, ferait retour dans la réalité sous forme de violence. La haine vitale est, à l'inverse, sublimée et mise ainsi au service de la construction du sujet et du lien mère/enfant, dans lequel ce dernier serait délogé de sa place de phallus imaginaire, et la mère constituée comme sujet pour l'enfant alors soumis à la loi.

C'est quand même au sein de ce temps "idyllique " de " préoccupation maternelle primaire " que Winnicott avance que " la mère hait son petit enfant dès le début... ". Façon de souligner «le dilemme de la fonction maternelle » (J. Aubry), cette difficulté et cette extrême nécessité de se séparer d'un objet qui nous est vitalement dépendant. Le premier mouvement allant à contre-courant du second, la question ne peut se résoudre que dans le compromis maternel sous la forme de l'ambivalence permettant de soutenir à la fois une position de mère et une position de femme. Difficile de ne pas se penser " toute " quand on est indispensable et de ne pas laisser s'écraser la féminité sous la maternité.

Pas plus que l'enfant ne se console un jour de l'insuffisance du sein maternel, la mère ne peut se trouver satisfaite à la mesure de l'amour de l'objet. C'est pourquoi ils se séparent. Personne n'a comblé personne, personne n'est demeuré dans le leurre et l'illusion qu'avec du deux on fait du un, c'est-àdire dans ce lieu mortifere, cette rencontre du réel, la jouissance, l'au-delà du principe de plaisir, avant le sujet, avant la vie, lieu au sein duquel nul écart ne verra s'inscrire et se réinscrire le désir singulier. Avec du deux, on fait d'emblée du trois.

On retrouve la notion de haine originaire que Freud ne faisait pas ressortir de l'affect ou de l'Imaginaire, mais d'un processus incontournable qui construit l'objet sous l'égide de l'opposition plaisir/déplaisir. Au regard du mouvement 
de séparation, cette haine originaire prend une dimension symbolique.

Ici, grâce au mécanisme de renversement de la pulsion en son contraire que Freud décrit comme un des destins possibles des pulsions, l'ambivalence maternelle reposerait non plus sur la transformation de l'amour en haine mais sur la transformation de la motion hostile en motion tendre.

L'amour maternel sans ambivalence voit s'abolir les distances et surgir un amour total, premier, meurtrier. En nommant ce qui de la haine s'inscrit d'emblée dans cet amour, la question de la vie et de la mort peut s'inscrire dans le symbolique au lieu d'être mortifere. Inscription concomitante du temps où le père réel réalise l'écart en offrant à l'enfant les signifiants du père symbolique, de la loi, celle qui interdit tout retour en arrière, au réel premier.

Que nous utilisions le vécu infantile apocalyptique que décrit M. Klein ("la peur de l'enfant d'être dévoré, dépecé, déchiré, sa terreur d'être entouré et poursuivi par des personnages menaçants »), ou les conflits et complexes freudiens, du point de vue de l'enfant et du point de vue de la mère, c'est un vécu destructeur qu'il faut symboliser.

La clinique du nourrisson témoigne parfois de ces regards maternels vides qui entraînent le désarroi chaotique chez le bébé alors vraisemblablement submergé par la pulsion de mort. Peut-être est-ce ce que recouvre l'" aliénation déshumanisante " que Lacan évoque dans son Séminaire Les psychoses et relevant d'un archaïque et terrifiant "surmoi maternel » qui inhibe les pulsions de vie.

Plus spectaculaire, l'infanticide témoigne d'une résurgence du meurtre dans le réel, absence impossible à penser dans un lieu autre que celui de la destruction et de la mort où la mère pourra se prétendre, telle Médée, une et toute. Médée pour qui la mort est moins terrifiante que l'intolérable castration. 


\section{CONCLUSION}

Que la haine ne s'engage pas ou qu'elle s'engage dans l'excès, au mouvement psychique resterait confondu son pur effet meurtrier repérable dans les comportements maternels de toute-puissance. Médée, narcissiquement blessée, se veut plus puissante que le père et confond rivalité imaginaire et pulsion de mort. (Elle était toujours enceinte.)

Laissons Rimbaud conclure :

"Et la mère, fermant le livre du devoir,

S'en allait satisfaite et très fière, sans voir,

Dans les yeux bleus et sous le front plein d'éminences,

L'âme de son enfant livrée aux répugnances." 